\title{
Photochemical processes in doped argon-neon core-shell clusters: The effect of cage size on the dissociation of molecular oxygen
}

\author{
T. Laarmann ${ }^{\text {a) }}$ \\ Max-Born-Institut, Max-Born Strasse 2a, D-12489 Berlin, Germany \\ H. Wabnitz \\ Hamburger Synchrotronstrahlungslabor HASYLAB at Deutsches Elektronen-Synchrotron DESY, \\ Notkestrasse 85, D-22603 Hamburg, Germany \\ K. von Haeften \\ Department of Physics and Astronomy, University of Leicester, Leicester LE1 7RH, Great Britain \\ T. Möller \\ Technische Universität Berlin, Hardenbergstrasse 36, D-10623 Berlin, Germany
}

(Received 19 September 2007; accepted 29 October 2007; published online 2 January 2008)

\begin{abstract}
The caging effect of the host environment on photochemical reactions of molecular oxygen is investigated using monochromatic synchrotron radiation and spectrally resolved fluorescence. Oxygen doped clusters are formed by coexpansion of argon and oxygen, by pickup of molecular oxygen or by multiple pickup of argon and oxygen by neon clusters. Sequential pickup provides radially ordered core-shell structures in which a central oxygen molecule is surrounded by argon layers of variable thickness inside large neon clusters. Pure argon and core-shell argon-neon clusters excited with $\sim 12 \mathrm{eV}$ monochromatic synchrotron radiation show strong fluorescence in the vacuum ultraviolet (vuv) spectral range. When the clusters are doped with $\mathrm{O}_{2}$, fluorescence in the visible (vis) spectral range is observed and the vuv radiation is found to be quenched. Energy-resolved vis fluorescence spectra show the $2{ }^{1} \Sigma^{+} \rightarrow 1{ }^{1} \Sigma^{+}\left(\operatorname{ArO}\left({ }^{1} S\right) \rightarrow \operatorname{ArO}\left({ }^{1} D\right)\right)$ transition from argon oxide as well as the vibrational progression $A^{13} \Delta_{u}\left(\nu^{\prime}=0\right) \rightarrow X^{3} \Sigma_{g}^{-}\left(\nu^{\prime \prime}\right)$ of $\mathrm{O}_{2}$ indicating that molecular oxygen dissociates and occasionally recombines depending on the experimental conditions. Both the emission from $\mathrm{ArO}$ and $\mathrm{O}_{2}$ as well the vuv quenching by oxygen are found to depend on the excitation energy, providing evidence that the energy transfer from the photoexcited cluster to the embedded oxygen proceeds via the $\mathrm{O}_{2}^{+}$ground state. The $\mathrm{O}_{2}^{+}$decays via dissociative recombination and either reacts with Ar resulting in electronically excited $\mathrm{ArO}$ or it recombines to $\mathrm{O}_{2}$ within the $\mathrm{Ar}$ cage. Variation of the Ar layer thickness in $\mathrm{O}_{2}-\mathrm{Ar}-\mathrm{Ne}$ core-shell clusters shows that a stable cage is formed by two solvation layers. (C) 2008 American Institute of Physics.
\end{abstract}

[DOI: $10.1063 / 1.2815798]$

\section{INTRODUCTION}

The unimolecular dissociation of a diatomic molecule in the gas phase belongs to one of the best understood type of chemical reactions. Most chemical reactions in nature, however, take place in condensed matter, either at surfaces, interfaces, or in liquids. These different environments modify the potential energy surface, they affect the intramolecular energy redistribution, or they cause steric effects where the ability of the molecule to undergo structural changes is inhibited by the lack of space.

In the last decade, the so-called "cage-effect" of the matrix material on a molecule has triggered a lot of theoretical and experimental work related to matrix isolation spectroscopy. ${ }^{1-6}$ The matrix atoms act like a cage and prevent the dissociation products breaking through the first solvation shell. As a consequence the dissociation of a molecule

\footnotetext{
${ }^{a)}$ Present address: Hamburger Synchrotronstrahlungslabor HASYLAB at Deutsches Elektronen-Synchrotron DESY, Notkestrasse 85, D-22603 Hamburg, Germany. Electronic mail: laarmann@mbi-berlin.de.
}

may be completely suppressed. The suppression of dissociation will depend on a variety of factors, such as the excess energy of the molecular reaction, the conversion efficiency into kinetic energy of the products, and the bond strength of the solvation layers. Thus, the systematic investigation of the cage effect is of great interest and will ultimately contribute to a comprehensive understanding of the role of the host environment on chemical reactions. Further, it is an interesting question whether photochemical processes of molecules embedded inside a "soft" matrix could be controlled by coating them with a well-defined number of atoms of a third material. More specifically, how many cage atoms are needed to effectively suppress dissociation? Finally, the dissociation or fragmentation of molecules plays a significant role in mass spectrometry, which often makes chemical analysis difficult. Here, deliberate caging of molecules could provide a route to suppress fragmentation and hence facilitate the assignment of mass peaks.

A recent review on the ultrafast dynamics of halogens in rare gas solids has highlighted the effects of the different 
guest sites, the orientation of the molecule with respect to the crystal, and its vibrational and rotational motion on its probability to dissociate. ${ }^{7}$ Molecules that assume single substitutional sites in cryogenic solid Ar are likely to dissociate if the molecular axis points towards a hole in the fcc crystal. Molecules in double substitutional sites with cylindrical symmetry are forced to vibrate like a piston against an atom in the cage. In this case dissociation is effectively inhibited. Where a dissociating molecule is able to exit the cage, it is thought to do so via interstitial binding sites of the products because the cryogenic crystalline matrix does not allow for structural rearrangements.

The restrictions found for the molecular dynamics associated with cryogenic lattices are relaxed for solution where molecules have one or more solvation layers. Here, the cage exit should be possible by simply breaking bonds within the solvation layer, the likelyhood of which should depend on the bond strength and the number of solvation layers. Because matrix studies offer limited flexibility to investigate the effect of the thickness of the solvation layer, studies on doped clusters in the gas phase have been undertaken using different spectroscopic techniques. ${ }^{8}$ Fluorescence spectroscopy is often used to identify the products of photochemical reactions in clusters, ${ }^{9-19}$ and femtosecond laser excitation provides information on the time evolution of reactions. ${ }^{20,21}$ Femtosecond photoelectron spectroscopy on mass selective iodine Ar anion clusters showed that a solvation shell of 20 Ar atoms was sufficient to induce recombination of the iodine fragments ${ }^{21,20}$ and similar experiments on iodine in neutral Ar clusters revealed that caging was completed within $10^{-12} \mathrm{~s}^{22-24}$ A thorough investigation of the cage effect of hydrogen halides doped into the interior or onto the surface of clusters and aligned in a static electric and a laser field was recently undertaken by Buck and co-workers. ${ }^{25-30}$ They measured the kinetic energy of the hydrogen atom as a function of the orientation and showed that the dissociation of hydrogen halides can even be inhibited on the cluster surface because the light hydrogen carries almost all the kinetic energy and is caged by the cluster and the heavy halogen atom.

Another extreme form of a matrix cage is liquid helium. Droplets of liquid helium provide the softest matrix material in nature, and surprisingly even for this extreme case caging is observed in the fragmentation upon electron impact ionization. ${ }^{31,32}$ However, here the observation of intact cluster ions of the embedded molecular clusters was attributed to efficient removal of the excess energy due to rapid cooling by the superfluid helium.

In this paper, we have investigated the cage effect on the dissociation of photoexcited molecular oxygen. For this purpose we have doped diatomic oxygen molecules into rare gas clusters and varied the thickness of the solvation layer. The clusters were excited with monochromatic synchrotron radiation and the emitted light was detected by integral and energy-resolved fluorescence spectroscopy in different wavelength ranges. These emission spectra allowed us to identify different photochemical reaction products of the oxygen and provided a detailed insight into the reaction pathway. Our primary objective was to investigate how many solvation layers are needed to inhibit dissociation. Our results show that recombination of photofragmented $\mathrm{O}_{2}$ becomes possible when there are two solvation layers of argon. Less than two solvation layers (50 atoms) do not affect dissociation, whereas the deposition of more than 100 atoms completely cages the oxygen molecule in the cluster beam. In contrast, solvation layers of neon were found to be unable to suppress the dissociation of $\mathrm{O}_{2}$.

The paper is organized in the following way. In Sec. II we explain the methodology in greater detail, and in Sec. III we give a description of the experimental setup. Particularly, we focus on the preparation of shell-like structures using the so-called sequential pickup technique. The experimental results are presented and discussed in Sec. IV. We first discuss the main relaxation channels of large $\mathrm{Ar}_{9700}$ clusters doped with $\mathrm{O}_{2}$ molecules in a coexpansion of a dilute $\mathrm{Ar} / \mathrm{O}_{2}$ gas mixture (Sec. IV A), before focussing on the pickup experiments with embedded $\mathrm{O}_{2}$ molecules surrounded by a welldefined number of Ar atoms $N, 50 \leqslant N \leqslant 100$ (Sec. IV B). Section $\mathrm{V}$ summarizes our findings.

\section{METHODOLOGY}

\section{A. General remarks}

We have chosen oxygen because $\mathrm{O}_{2}^{+}$is known to undergo dissociative recombination in the gas phase (see Ref. 33, and references therein). Here, the matrix cage comes into play because the photoelectron clearly has an increased chance to recombine with $\mathrm{O}_{2}^{+}$due to backscattering in the matrix. The matrix cage will also increase the dissociation threshold energy which can be detected by a decrease of the photodissociation yield. ${ }^{34}$ In the case of recombination of the photofragments of $\mathrm{O}_{2}$, the ${ }^{3} \Delta_{u}$ electronic level will be populated and visible (vis) radiative decay to the ${ }^{3} \Sigma_{g}^{-}$ground state will be observed. The fluorescence of this transition will be taken as an indicator for inhibited dissociation.

\section{B. Cluster preparation}

Experiments with clusters have several benefits such as a continuous sample renewal and simple preparation techniques. Rare gas clusters are particularly well suited as host material because of their low chemical reactivity. Furthermore, rare gas clusters are transparent over a wide range of energies in the vacuum ultraviolet (vuv). Central to our study are two different methods of preparing the doped clusters. The method of supersonic coexpansion of dilute amounts of $\mathrm{O}_{2}$ in argon into a vacuum produces clusters which on average are doped with a single molecule, depending on the concentration. The $\mathrm{O}_{2}$ molecule acts as a condensation nucleus and thus it is more likely to be found inside the clusters than on surface sites. We expect that this tendency is even greater for larger Ar clusters because the $\mathrm{Ar}-\mathrm{O}_{2}$ intermolecular interaction is more favorable than the Ar-Ar. Even so, $\mathrm{O}_{2}$ bound to the Ar cluster surface cannot be excluded, and to avoid this possibility this work produces doped core-shell clusters by the multiple pickup method employing two cross jets in series. The pickup method is well suited to dope solid clusters such as $\mathrm{SF}_{6}$ with molecules at the surface. ${ }^{35,36}$ If the cluster is liquid such as helium it is possible to assemble clusters inside them by multiple pickup, because the embed- 
ded particles collide with helium and eventually with themselves: thus they cool and agglomerate. ${ }^{37}$ The average size of the embedded clusters is determined by Poisson statistics and shows a well-defined dependence on the density of the pickup gas. ${ }^{38} \mathrm{~A}$ similar behavior is observed for hydrogen clusters which were found to be liquid on their surface. Collisions with two $\mathrm{Xe}$ atoms on average led to the formation of Xe dimers inside the hydrogen clusters. ${ }^{39}$ Clusters of neon where found to be liquid when smaller than 150 atoms, ${ }^{40}$ whereas larger neon clusters still remain quite soft and possess a liquidlike surface ${ }^{41}$ because of their low binding energy compared to other substances. Multiple doping usually leads to the formation of embedded clusters, because atoms can diffuse easily through the neon matrix within the time scales of the experiments (microsecond to millisecond). Since the binding energy increases towards the center of a cluster, the embedded clusters are preferentially located in the center. In extreme cases, multiple doping can lead to embedded clusters even in harder materials such as $\mathrm{Ar}$ clusters. ${ }^{42,43}$ Fast multiple collisions heat the cluster increasing the dopant mobility before the system can relax by evaporative cooling. Depending on the experimental conditions, it is possible that only a few Ar atoms remain attached to a cluster formed by pickup of embedded atoms. The size of these embedded clusters depends on the dopant gas density in the pickup region as for the helium droplets and is given by Poisson functions. ${ }^{44}$ Molecular dynamics simulations have shown that the doping site in Ar clusters depends on the binding energy and the size of the dopant. ${ }^{36}$ Further simulations on single and multiple doping of Ar clusters investigated the structural rearrangements and their time evolution in more detail ${ }^{45,46}$ and provided results that agreed with the experimental findings by Rutzen et al. ${ }^{42}$

Owing to recent progress in the multiple pickup preparation techniques, it is now possible to cover the surface of embedded molecules or clusters with a well-defined number of atoms of a third material. ${ }^{47}$ These atoms form a shell around the impurity on the inside of large host clusters allowing the controlled modification of surface properties of the embedded system and providing information on, for instance, hindered desorption or dissociation processes at interfaces. Employing this novel technique, it recently became possible to observe electronically excited $\mathrm{Ar}$ atoms in ArKrNe core-shell clusters. ${ }^{47}$ It was even possible to track the diffusion of excited $\mathrm{Ar}$ atoms through a cage of $\mathrm{Kr}$ and successive $\mathrm{Ne}$ atoms and finally to identify them being desorbed from the cluster surface. ${ }^{47}$ The method of cluster aggregation has also beeen applied to other types of materials, such as metals or semiconductors: as an example, Ag clusters inside Ar clusters have recently been produced. ${ }^{48}$

\section{Spectroscopic characterization of photoreactions}

In the present experiments argon as the cage material also serves as an optical sensitizer because direct optical excitation of $\mathrm{O}_{2}$ has a comparatively low cross section and therefore could not be detected. The electronic excitations of Ar clusters are well understood in terms of surface and bulk excitons. ${ }^{49}$ The excitons exhibit discrete bands with well known energies which overlap with the levels of $\mathrm{O}_{2}^{+}$. Whereas in pure Ar clusters the energy dissipation process leads mainly to vuv fluorescence from atomic and molecular self-trapped excitons ( $a$-STE and $m$-STE) ${ }^{50,51}$ the energy relaxation pathways may change dramatically when clusters are doped with impurities. We employed two spectrally integrating detectors: (i) one that was sensitive in the vuv and therefore solely measured the fluorescence of pure Ar clusters and (ii) one sensitive to visible light, thus detecting only the fluorescence from oxygen related photoreaction products. There was almost no spectral overlap between the two detectors $^{52}$ and hence the ratio between the signals could be used to measure the extent to which a photoreaction has taken place. With a third detector we spectrally resolved the fluorescence at fixed excitation wavelengths which gives a measure of the abundance of the $\mathrm{ArO}$ and $\mathrm{O}_{2}$ reaction products.

A specific reason for the choice of the $\mathrm{O}_{2} / \mathrm{Ar}$ system in the present study is that some of its features considerably facilitate the interpretation of the spectra. Van-der-Waals bonded $\mathrm{O}_{2}$ dimers and oligomers decay predominantly via nonradiative channels upon photoexcitation in the relevant energy range $(\approx 12 \mathrm{eV}) .{ }^{53,54}$ This property is quite useful for optimizing the experimental conditions for single molecule doping. Another important property of oxygen is that photoexcited-free $\mathrm{O}_{2}$ does not emit in the time frame of the present experiment. Thus, the observation of oxygen related emission indicates that a photoreaction of a single molecule affected by the host environment must have occurred. On the other hand, it is not possible to identify different oxygen dopant sites by direct excitation of the molecules, such as has been done for $\mathrm{Xe}$ atoms in various types of rare gas clusters. ${ }^{55-57}$ Finally it is worth noting that the vuv fluorescence yield of pure Ar clusters is close to unity. ${ }^{58}$ This is important here since we will take the quenching of the Ar emission as a measure for the transfer of energy to the oxygen and the activation of the photoreaction.

\section{EXPERIMENT}

The measurements were performed at the experimental station CLULU (cluster luminescence) at the Hamburg Synchrotron Radiation Laboratory (HASYLAB). The basic experimental setup is described in detail in Ref. 58. For the current investigation only a few modifications to the experimental apparatus have been made. These concern the fluorescence light detectors and the multiple pickup source for the production of core-shell clusters, for which we have employed two consecutive cross beams (Fig. 1).

Briefly, core-shell clusters were produced as follows: in a first step intense beams of $\mathrm{Ne}_{n}$ clusters were prepared in a supersonic expansion using a conical nozzle which had a throttle diameter of $d=200 \mu \mathrm{m}$, a half-opening angle of $\alpha=4^{\circ}$, and which was cooled with liquid helium. The average cluster size was estimated using well established scaling laws. For instance, at a nozzle temperature of $T_{0}=30 \mathrm{~K}$ and a stagnation pressure of $p_{0}=200$ mbars, the average Ne cluster size was determined as approximately $n=7500 .{ }^{59,60}$ The full width at half maximum $\Delta N$ of the cluster size distribution 


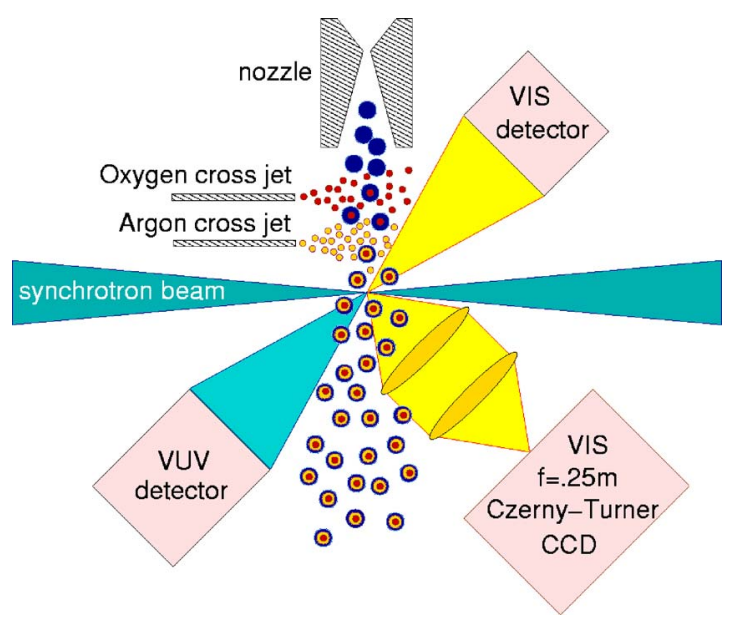

FIG. 1. (Color online) Schematics of the experimental setup. Single oxygen molecules are doped to neon clusters by the pickup method using a cross jet. A second cross jet thereafter dopes the clusters with argon. The argon atoms form shells around the oxygen. The doped clusters are excited by monochromatic synchrotron radiation. The fluorescence is recorded in different wavelength regions. Dispersed fluorescence is recorded using a $f=0.25 \mathrm{~m}$ Czerny-Turner spectrometer equipped with a liquid nitrogen cooled CCD camera.

was approximately $N$. Next, $\mathrm{O}_{2}$ molecules from a cross jet were picked up by the Ne clusters. We assume that the collision with $\mathrm{O}_{2}$ softens the $\mathrm{Ne}$ cluster and hence the $\mathrm{O}_{2}$ molecules are able to penetrate deep into the cluster. This behavior is expected because of the liquid cluster surface, the increased mobility of the molecules in the heated cluster, and also because of the polarization drag force towards the center. $^{41}$ Molecular dynamics simulations on Ar clusters that collide with single $\mathrm{Kr}$ atoms show that a single collision changes the cluster structure so that it becomes amorphous. ${ }^{45}$ They further show that within the time scale of the experiment most of the $\mathrm{Kr}$ atoms become completely solvated by the Ar. ${ }^{45}$ For the much softer Ne clusters the tendency of the dopant to move towards the center should be even stronger.

In the final step the $\mathrm{O}_{2}$ doped $\mathrm{Ne}_{7500}$ cluster is codoped with up to $100 \mathrm{Ar}$ atoms. As $\mathrm{O}_{2}$ and Ar have similar interaction potentials with $\mathrm{Ne}$, we assume that the Ar atoms are also incorporated inside the Ne cluster. This process should be facilitated by the multiple collisions which heat up the $\mathrm{Ne}$ cluster on a short time scale. The Ar atoms are therefore expected to locate in the direct neighborhood of $\mathrm{O}_{2}$, and depending on the total number of embedded Ar atoms, it is likely that the oxygen becomes completely surrounded. The core-shell $\mathrm{O}_{2}-\mathrm{Ar}_{N}$ structure will stabilize inside the Ne host cluster as it will relax to its equilibrium temperature of $10 \mathrm{~K}$ by evaporative cooling of $\mathrm{Ne}$ atoms. ${ }^{61}$ Control of the crossjet densities allows $\mathrm{O}_{2} \mathrm{Ar}_{N}$ clusters to be produced with 50 $\leqslant N \leqslant 100$ Ar atoms inside host Ne clusters. ${ }^{62}$ The formation of embedded clusters is finished before the clusters interact with synchrotron radiation ${ }^{63}$ because the structural rearrangements are fast ${ }^{45}$ compared to the flight time of the clusters $\left(\approx 4 \times 10^{-5} \mathrm{~s}\right)$.

For the pickup method, the interaction potential between host cluster atoms and the impurity is of fundamental importance. The technique only works if the binding energy of the cluster atoms is significantly smaller than the interaction be-
TABLE I. Lennard-Jones potential depth $\epsilon$ of different homo- and heteronuclear molecules (Ref. 64). The parameter $\epsilon^{\prime}=\epsilon / \epsilon_{\mathrm{Ne}-\mathrm{Ne}}$ describes the strengths of different bindings relative to the $\mathrm{Ne}-\mathrm{Ne}$ interaction

\begin{tabular}{ccc}
\hline \hline Molecule & $\epsilon(\mathrm{meV})$ & $\epsilon^{\prime}\left(\epsilon_{\mathrm{Ne}-\mathrm{Ne}}\right)$ \\
\hline $\mathrm{Ne}-\mathrm{Ne}$ & 3.19 & 1 \\
$\mathrm{Ar}-\mathrm{Ar}$ & 10.34 & 3.24 \\
$\mathrm{O}_{2}-\mathrm{O}_{2}$ & 22.32 & 7.0 \\
$\mathrm{Ne}-\mathrm{Ar}$ & 5.74 & 1.80 \\
$\mathrm{Ne}-\mathrm{O}_{2}$ & 8.44 & 2.65 \\
$\mathrm{Ar}-\mathrm{O}_{2}$ & 15.19 & 4.76 \\
\hline
\end{tabular}

tween the impurity and the cluster, otherwise the dopant atoms or molecules cannot stick to the clusters surface (or even if they did they would evaporate after a very short time ${ }^{45}$ ). The depth $\epsilon$ of different homonuclear Lennard-Jones pair potentials of rare gas atoms ${ }^{64}$ and molecular oxygen ${ }^{65,66}$ summarized in Table I is a good guide to compare different bond strengths. Zero-point motion can be neglected. The parameters for heteronuclear pair potentials (e.g., $\mathrm{Rg}, \cdots \mathrm{O}_{2}$ ) were derived by using the standard procedure, ${ }^{64} \epsilon_{\mathrm{Rg} \cdots \mathrm{O}_{2}}$ $=\left(\epsilon_{\mathrm{Rg}} \epsilon_{\mathrm{O}_{2}}\right)^{1 / 2}$. The value of $\epsilon^{\prime}=\epsilon / \epsilon_{\mathrm{Ne}-\mathrm{Ne}}$ is a measure of the strengths of different bindings relative to the $\mathrm{Ne}-\mathrm{Ne}$ interaction. According to the values in Table I, both molecular oxygen and Ar atoms stick to the Ne cluster surface and are thus expected to penetrate towards the inside during the cluster melting. The sizes of the embedded clusters in our experiments were determined using theoretical and experimental work by Lewerenz et al. ${ }^{63}$ and our previous work. ${ }^{47}$ The average number of atoms pickup depends mainly on the number of collisions between the Ne clusters and the $\mathrm{O}_{2}$, and it is assumed that almost all encounters lead to $\mathrm{O}_{2}$ molecules becoming trapped by the clusters. Note that all numbers given below are mean values for the cluster sizes with an estimated error for the embedded species of $\approx 20 \%$. In the present work, special care was taken in order to avoid multiple $\mathrm{O}_{2}$ doping in the first pickup zone by keeping the density of molecular oxygen in the cross jet as low as possible.

$\mathrm{O}_{2}$ doped Ar clusters were also produced by the coexpansion method. This technique has previously been used with considerable success to produce $\mathrm{Xe}$ and $\mathrm{Kr}$ doped rare gas clusters over a large size range. ${ }^{40,55-57}$ In these studies it was possible to spectroscopically identify single or multiple doping and to relate these to the gas concentrations. These data provide the basis for the present work. For the coexpansion experiments in this work we produced $\mathrm{Ar}_{9700}$ clusters from a dilute gas mixture containing $1 \% \mathrm{O}_{2}$ in Ar at a stagnation pressure of $p_{0}=2500$ mbars, a nozzle temperature $T_{0}=105 \mathrm{~K}$, and using a conical nozzle with $d=100 \mu \mathrm{m}$ diameter and $\alpha=15^{\circ}$ half-opening cone angle. The previous work on $\mathrm{Xe}$ doped $\mathrm{Ar}$ clusters at this size showed that roughly $75 \%$ of the dopants reside in interior sites of the cluster.

Spectra were recorded by focusing monochromatic synchrotron radiation at a distance of $10 \mathrm{~mm}$ downstream from the nozzle onto the cluster beam. The synchrotron radiation was scanned between $11.5-12.9 \mathrm{eV}$ at a resolution of $2.5 \AA$ $(\approx 30 \mathrm{meV})$. This energy range is characterized by strong $\mathrm{Ar}$ cluster absorption (excitons). The fluorescence light emitted 


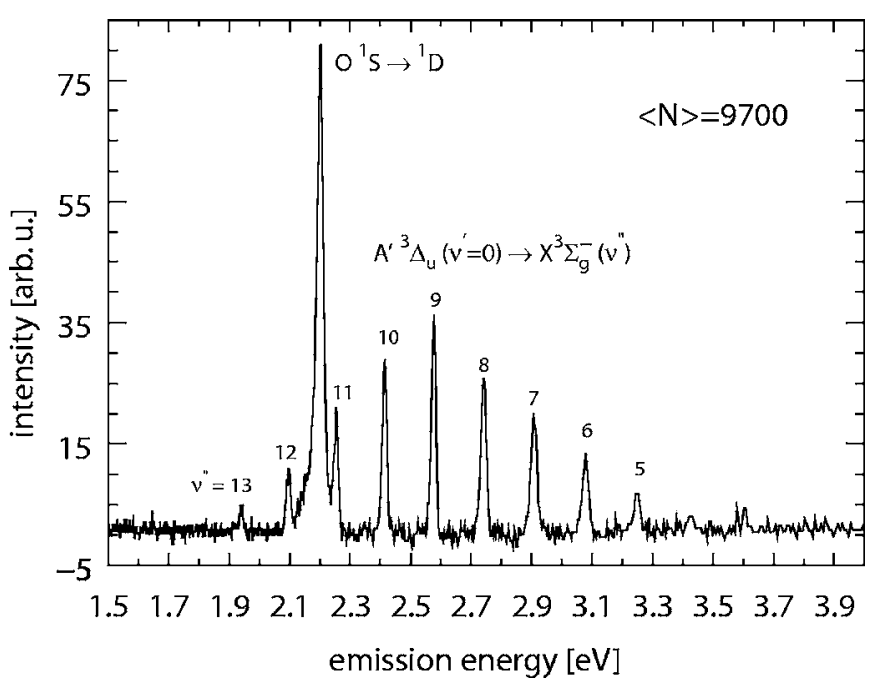

FIG. 2. Energy-resolved vis fluorescence of doped $\mathrm{O}_{2} \mathrm{Ar}_{9700}$ clusters upon excitation of the argon 1'l exciton at $12.38 \mathrm{eV}$. Radiative transitions of ArO $\quad 2^{1} \Sigma^{+} \rightarrow 1{ }^{1} \Sigma^{+}\left(\mathrm{O}\left({ }^{1} S\right) \rightarrow \mathrm{O}\left({ }^{1} D\right)\right)$ and the vibrational progression $A^{\prime 3} \Delta_{u} \rightarrow X^{3} \Sigma_{g}^{-}$of $\mathrm{O}_{2}$ are indicated. (Ref. 54).

by the doped clusters was detected by photomultipliers sensitive to different spectral ranges. The vuv fluorescence in the coexpansion experiments was recorded by a Valvo XP $2020(4-40 \mathrm{eV})$, covered with a thin layer of sodium salicylate to convert the vuv photons into visible photons. For the experimentally more demanding pickup studies, a "solarblind" Hamamatsu R 1460 photomultiplier (4.1-11.3 eV) was used. The low-energy fluorescence was measured with a Hamamatsu R 943-02 photomultiplier which is sensitive between $1.3-7.7 \mathrm{eV}$. The R 943-02 was Peltier cooled to suppress the dark count rate to a few counts per second. The integrated fluorescence excitation spectra were recorded by the photon counting techniques and were normalized for the spectral transmission of the synchrotron beamline and the beam current. In addition, spectral dispersed vis fluorescence at fixed excitation wavelength was measured using a CzernyTurner $f=0.25 \mathrm{~m}$ spectrometer and recorded by a liquid nitrogen cooled charge coupled device (CCD) camera. Background noise created by cosmic particles impinging on the CCD was removed using home-written software. ${ }^{67}$ The spectrometer was equipped with a 150 grooves/mm grating having a spectral resolution of $\approx 1 \mathrm{meV}$ at $1.5 \mathrm{eV}$. It was coupled to the experimental chamber via a lens system consisting of two LiF/suprasil achromats in order to minimize chromatic and spherical aberrations (see Fig. 1).

\section{RESULTS AND DISCUSSION}

\section{A. Coexpansion experiments}

Figure 2 shows an energy-resolved vis fluorescence spectrum of $\mathrm{O}_{2}$ doped $\mathrm{Ar}_{9700}$ clusters produced by the coexpansion of a $1 \%$ oxygen-argon gas mixture. The density of doped clusters in the intersection point with the synchrotron radiation is comparatively low and therefore the exposure time was $60 \mathrm{~min}$. The excitation energy was $12.38 \mathrm{eV}$, which corresponds to the longitudinal branch of the $n=1^{\prime} \mathrm{Ar}$ bulk exciton, where the prime denotes the spin orbit state $j=\frac{1}{2}$.
The sharp lines of the emission spectrum in Fig. 2 were assigned to the ArO transition $2{ }^{1} \Sigma^{+} \mathrm{O}\left({ }^{1} S\right) \rightarrow 1{ }^{1} \Sigma^{+} \mathrm{O}\left({ }^{1} D\right)$ and to the $\left(v^{\prime}=0\right) A^{\prime}{ }^{3} \Delta_{u} \rightarrow\left(v^{\prime \prime}=3-13\right) X^{3} \Sigma_{g}^{-}$of $\mathrm{O}_{2}$ vibronic progression $^{54}$ which is also known as the Herzberg III band. ${ }^{68}$ The Herzberg III band is forbidden for free molecules by dipole-radiation selection rules and is therefore extremely weak, ${ }^{3}$ however, in a matrix environment the Herzberg III band emission is known to be enhanced by several orders of magnitude and so can serve as a sensitive probe of local interactions and dynamics (see, e.g., Refs. 1-4 and references therein). Its presence shows that electronically excited oxygen molecules are embedded in the interior of argon clusters. Moreover, this emission is not seen from $\mathrm{O}_{2}$ dimers, ${ }^{53,54}$ giving evidence that our clusters are doped with single molecules.

The observed fluorescence line at $2.2 \mathrm{eV}$ is close in energy to the $\mathrm{O}\left({ }^{1} S\right) \rightarrow \mathrm{O}\left({ }^{1} D\right)$ transition of atomic oxygen at $2.22 \mathrm{eV}$. This atomic transition is dipole forbidden in the gas phase and has a long radiative lifetime of $\approx 0.71 \mathrm{~s} .{ }^{69} \mathrm{We}$ can therefore exclude the possibility that it stems from free oxygen or from dissociated excited oxygen that has been desorbed from the cluster surface. To some extent $\mathrm{O}\left({ }^{1} S\right)$ must remain in the cluster environment where it reacts with the Ar. Hence, the origin of the peak is electronically excited ArO (or $\operatorname{Ar}_{N} \mathrm{O}$ ) which has a shallow potential minimum in the excited state, as depicted in Fig. 4. The formation of $\mathrm{ArO}$ excimers in their $\left({ }^{1} S\right)$ state and their radiative transition into the $\operatorname{ArO}\left({ }^{1} D\right)$ state has been studied extensively in the past, triggered by the idea of using rare gas oxides to build powerful lasers. ${ }^{70}$ Given our limited spectral resolution we cannot distinguish $\mathrm{ArO}$ from $\mathrm{Ar}_{N} \mathrm{O}$.

Next, we will investigate the dependence of the fluorescence on the photoexcitation energy. Figure 3 shows the fluorescence excitation spectrum recorded by the vuvsensitive detector for pure $\mathrm{Ar}_{9700}$ and $\mathrm{O}_{2}$ doped $\mathrm{Ar}_{9700}$ clusters. Both the features of the doped and bare clusters follow the spectral profile of the tightly bound excitons, proving evidence that the oxygen is excited via the Ar. However, the vuv spectrum is much weaker in intensity when the clusters are doped with oxygen. The opposite is found in the vis spectrum. Although pure Ar clusters do not emit photons in the visible spectral range, a very weak contribution is still observed, which we attribute to accidental detection of blue light from the sodium salicylate wavelength converter in front of the vuv detector. We find that the decrease of vuv fluorescence and similarily the enhancement of the vis fluorescence are energy dependent. The vis emission is only observed above $11.97 \mathrm{eV}$, which is close to the ionization energy of $\mathrm{O}_{2}$ of $12.071 \mathrm{eV} .^{71}$ A similar threshold of $11.92 \mathrm{eV}$ is found for the decrease of the vuv luminescence. We will see in the following that the change in fluorescence yield is caused by the transfer of energy to the ionic states of oxygen. Note that the observed thresholds also coincide with the energies of characteristic surface excitations $(<12 \mathrm{eV})$. Since roughly $25 \%$ of the oxygen molecules are in or on the cluster surface, Ar surface excitons are another possible origin for energy transfer to oxygen and the observed threshold could be interpreted by a site-dependent energy transfer. We will see later that, even so, the energy transfer from Ar to $\mathrm{O}_{2}$ is 


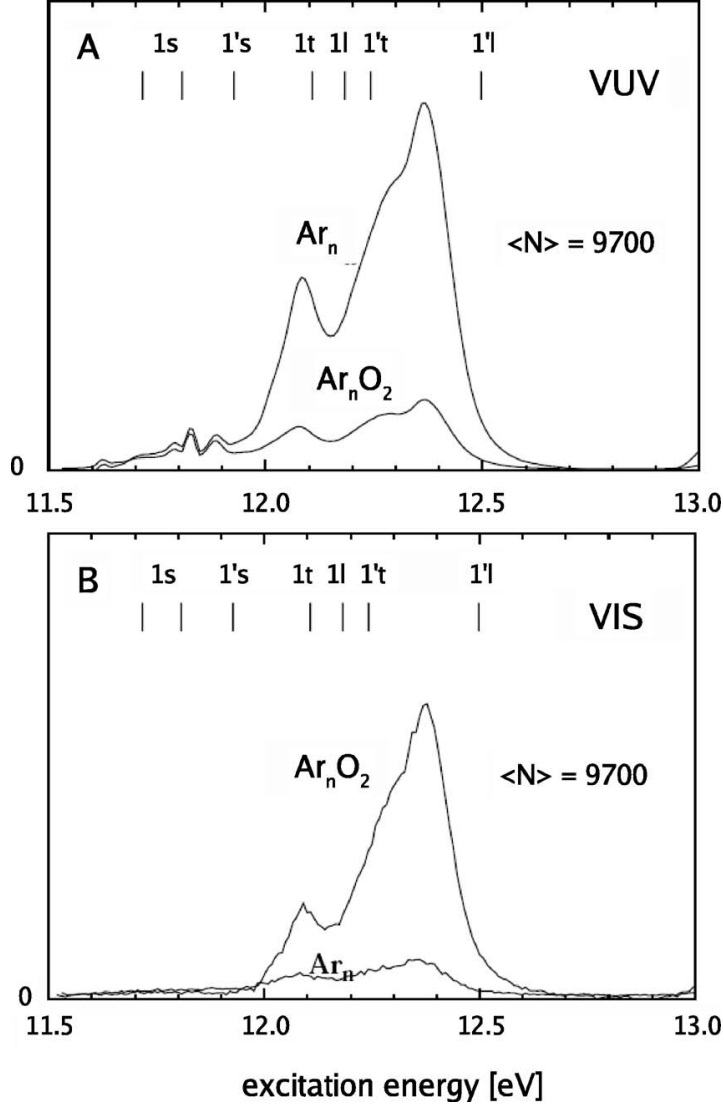

FIG. 3. Fluorescence excitation spectrum of pure $\mathrm{Ar}_{9700}$ and $\mathrm{O}_{2}$ doped $\mathrm{Ar}_{9700}$ clusters measured using the vuv-sensitive detector (a) and the vis sensitive detector (b). The surface excitons are labeled with $s$, and the bulk excitons with $t$ and $l$. The prime denotes the spin orbit state $j=\frac{1}{2}$. The fluorescence intensity is given in arbitrary units on a linear scale. The doped clusters were produced by $\mathrm{Ar} / \mathrm{O}_{2}$ coexpansion. not restricted to specific exciton sites and that the observed threshold is purely related to the onset of $\mathrm{O}_{2}$ ionization.

The energy transfer from $\mathrm{Ar}$ excitons to $\mathrm{O}_{2}^{+}$ions is followed by dissociative recombination along several repulsive curves as depicted in Fig. 4. The ${ }^{1} \Sigma_{u}^{+}$potential curve crosses with vibrational states of the molecular ion $(\nu=1,2)$ at $12.4 \mathrm{eV}$ and leads to the $\mathrm{O}\left({ }^{1} S\right)$ and $\mathrm{O}\left({ }^{1} D\right)$ states. ${ }^{33}$ Dissociation along this pathway produces photofragments with a kinetic energy of $\approx 0.4 \mathrm{eV}$.

$$
\operatorname{Ar}_{n}^{*} \mathrm{O}_{2} \rightarrow \mathrm{Ar}_{n} \mathrm{O}_{2}^{+}+e^{-} \rightarrow \operatorname{ArO}\left({ }^{1} S\right)+\mathrm{O}\left({ }^{1} D\right) .
$$

The dissociation of $\mathrm{O}_{2}$ along the $1{ }^{1} \Sigma_{u}^{+}$is a well known source of electronically excited atomic oxygen in the upper atmosphere and is responsible for the long-lived green light emission. Guberman has calculated the rate constants for these reaction, considering high Rydberg states and spin orbit induced state mixing, ${ }^{72}$ and found that a neutral Rydberg state $(n=7)$ as an intermediate step is involved,

$$
\mathrm{O}_{2}^{+} e^{-} \rightarrow \mathrm{O}_{2}(\mathrm{Ry}) \rightarrow \mathrm{O}+\mathrm{O} .
$$

In the present experiment it is possible that the argon excitons couple directly to the $\mathrm{O}_{2}$ Rydberg states, in fact the threshold being slightly lower than the ionization energy of the free molecule indicates that Rydberg states might be involved. We nevertheless have to emphasize that according to the lack of space for orthogonal wave functions in rare gas matrices impurity Rydberg states are expected to be strongly mixed and shifted in energy. ${ }^{73}$

It is important to note that the $\mathrm{ArO}$ excimer emission is only possible if both dissociation products are well separated from each other, for instance in different Ar shells, because recombination of the $\mathrm{O}$ atom pair is highly efficient in the $\mathrm{Ar}$ matrix. ${ }^{54} 1{ }^{1} \Sigma_{u}^{+}$photofragments that remain inside the same

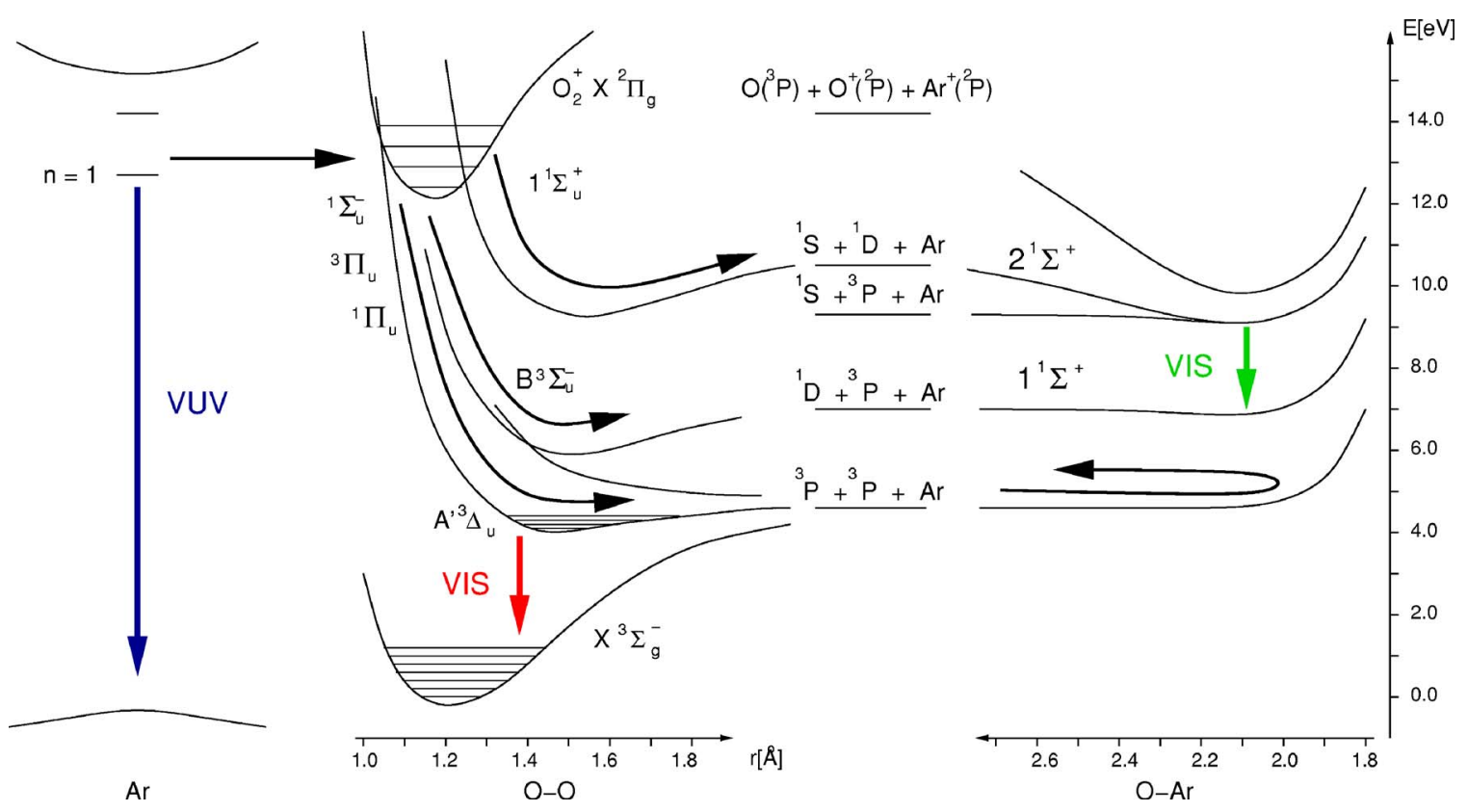

FIG. 4. (Color online) Schematics of the energy levels of the Ar excitons and the relevant oxygen and Ar-O potential curves. The relaxation pathways are indicated by arrows. For simplicity not all the repulsive curves dissociating into the ${ }^{3} P$ state $\left({ }^{1} \Pi_{u},{ }^{3} \Pi_{u}\right.$, and $\left.{ }^{1} \Sigma_{u}^{-}\right)$are plotted. The curves are based on the data from several publications. (Refs. 72, 75, 76, and 78). 
cage can recombine, relax into the $A^{\prime}{ }^{3} \Delta_{u},\left(\nu^{\prime}=0\right)$ state, and eventually fluoresce into several vibrational levels $\nu^{\prime \prime}$ of the $X^{3} \Sigma_{g}^{-}$ground state (Fig. 2).

The main relaxation channel by which the excited oxygen dimer is formed is similar to the $\mathrm{ArO}$ excimer formation and involves dissociative recombination. In the first step energy is transferred from the free Ar exciton to the ionic $\mathrm{O}_{2}^{+}$ ground state, then fast nonradiative decay by dissociative recombination via the ${ }^{3} \Pi_{u},{ }^{1} \Pi_{u}$, or the ${ }^{1} \Sigma_{u}^{-}$potential curves populates the ${ }^{3} P$ state, and finally cage-induced recombination populates the $A^{\prime}{ }^{3} \Delta_{u}$,

$$
\begin{aligned}
\operatorname{Ar}_{n}^{*} \mathrm{O}_{2} & \rightarrow \operatorname{Ar}_{n} \mathrm{O}_{2}^{+}+e^{-} \rightarrow \operatorname{Ar}_{n}+2 \cdot \mathrm{O}\left({ }^{3} P\right) \\
& \rightarrow \operatorname{Ar}_{n} \mathrm{O}_{2}^{*}\left(A^{\prime}{ }^{3} \Delta_{u}\right) .
\end{aligned}
$$

Taking the dissociation energy of $\mathrm{O}_{2}\left(\mathrm{O}\left({ }^{3} P\right)\right)$ of $5.11 \mathrm{eV}$ and the excitation energy of $12.38 \mathrm{eV}$, the photofragments gain up to $3.5 \mathrm{eV}$ kinetic energy each. The nonradiative decay via the $B^{3} \Sigma_{u}^{-}$state yields identical products. Here, oxygen atoms $\mathrm{O}\left({ }^{1} D+{ }^{3} P\right)$ with more than $4 \mathrm{eV}$ kinetic energy are produced in the first step. The oxygen atoms recombine if caging occurs. Subsequent vibrational relaxation is followed by fast decay via a curve crossing towards the $\mathrm{O}\left({ }^{3} P+{ }^{3} P\right)$ states. A second caging event produces oxygen in the excited $A^{\prime}{ }^{3} \Delta_{u}$ state from which the vis fluorescence is observed.

We have also considered energy transfer from free Ar excitons into local electronically excited molecular centers $\left(\mathrm{Ar}_{2}^{*} \quad m \text {-self-trapped excitons }\right)^{51}$ and subsequent Förster-Dexter ${ }^{74}$ transfer to the $\mathrm{O}_{2}$,

$$
\operatorname{Ar}_{n}^{*} \mathrm{O}_{2} \rightarrow \operatorname{Ar}_{2}^{*} \operatorname{Ar}_{(n-2)} \mathrm{O}_{2} \rightarrow \operatorname{Ar}_{n} \mathrm{O}_{2}^{*} \text {. }
$$

Energy transfer from relaxed $\mathrm{Ar}_{2}^{*}$ self-trapped excitons would yield dissociation products with reduced kinetic energy of $\approx 2.3 \mathrm{eV}$, although this scenario is energetically possible, we believe that the first relaxation mechanism is the correct one. The main reason for this is that the vis emission only occurs when the Ar is excited above the ionization energy of molecular oxygen.

Figure 4 shows the energy levels of the Ar excitons, the relevant potential curves of $\mathrm{O}_{2}$ and $\mathrm{ArO}$, and the dominant relaxation pathways which are indicated by arrows. We would like to add that we found no signs of low-lying $\mathrm{Ar}_{2}^{+} \mathrm{O}^{-}$ charge transfer states being involved in the relaxation cascade of oxygen doped Ar clusters. These states would fluoresce in the UV between 4.5 and $7 \mathrm{eV}$, as known from spectroscopic studies in solid matrices. ${ }^{75,76}$ However, in small clusters the transition energies may slightly change because of charge delocalization. ${ }^{77}$ Our energy resolved-spectra do not show such emissions, at least for photon energies below $4.4 \mathrm{eV}$. Moreover, the decrease of the total vuv fluorescence yield recorded from Ar clusters upon doping (shown in Fig. 3) would not have been possible if charge transfer states were populated.

\section{B. Pickup experiments}

In the following section we will discuss how many Ar atoms are actually needed to hinder dissociation of molecular oxygen by either trapping the excited dissociation products inside different cages ( $\mathrm{ArO}$ emission) or inside the same

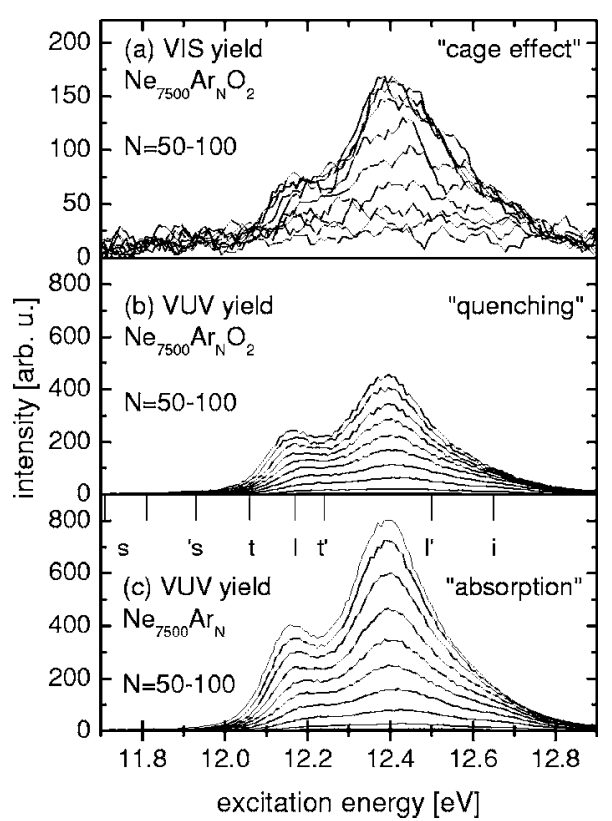

FIG. 5. Excitation spectra of vis fluorescence (a) and vuv fluorescence (b) of core-shell $\mathrm{Ne}_{7500} \mathrm{Ar}_{N} \mathrm{O}_{2}$ clusters are compared with vuv fluorescence excitation spectra of embedded Ar clusters containing no molecular oxygen (c). All spectra $[(\mathrm{a})-(\mathrm{c})]$ rise in intensity with increasing $N$, but the spectra in (b) are less pronounced than spectra in (c). $N$ denotes the number of Ar atoms. The clusters are excited in the energy range of characteristic Ar cluster absorption. Excitation energies of surface excitons $(s)$, longitudinal $(l)$ and transverse $(t)$ bulk excitons, and the NeAr interface absorption $(i)$ are indicated in the figure. The prime denotes the spin orbit state $j=\frac{1}{2}$. The spectra in (a) are quite noisy due to the low signal and therefore have to be averaged over five adjacent data points.

cage $\left(\mathrm{O}_{2}\right.$ emission). To address this question we have produced large Ne clusters that contain molecular oxygen covered with a well-defined number $N$ of coated Ar atoms (50 $\leqslant N \leqslant 100$ ) in their center. The vis and vuv excitation spectra of $\mathrm{Ne}_{7500} \mathrm{Ar}_{N} \mathrm{O}_{2}$ clusters produced in that way are shown in Fig. 5. The Ar layer contained $N=55,60,65, \ldots, 100$ atoms on average. The spectra in Fig. 5 are very similar to those of clusters produced by coexpansion (Fig. 3) and show the characteristic absorption of Ar excitons. However, the surface absorption band characteristic for free $\mathrm{Ar}$ clusters $(<12 \mathrm{eV})$ disappears when Ar clusters are embedded inside Ne. This observation is understandable because of direct interaction of the Ar excitons with the surrounding $\mathrm{Ne}$ atoms which gives rise to a new $\mathrm{Ar} / \mathrm{Ne}$ interface absorption band that appears as a shoulder around $12.65 \mathrm{eV} .^{79,80}$ The appearance of this band verifies that our initial assumption of complete solvation of the Ar shells inside the Ne cluster and site-independent energy transfer from excitons to $\mathrm{O}_{2}$ was correct. The Ar/Ne interface band lies above the $\mathrm{O}_{2}$ ionization threshold. All the spectra in Fig. 5 including the vis excitation spectra in Fig. 5(a) show this band, providing strong evidence for an energy transfer from the $\mathrm{Ar} / \mathrm{Ne}$ interface to the oxygen. This finding shows that whether excitons are located in the interior, at the surface, or at the interface of Ar clusters, it makes no difference to their ability to transfer energy to the oxygen. It is therefore clear that the observed energy dependence in Fig. 3 is purely related to the onset of dissociative recombination in the molecular oxygen ion.

By analyzing the fluorescence data in Figs. 5(a)-5(c) one 

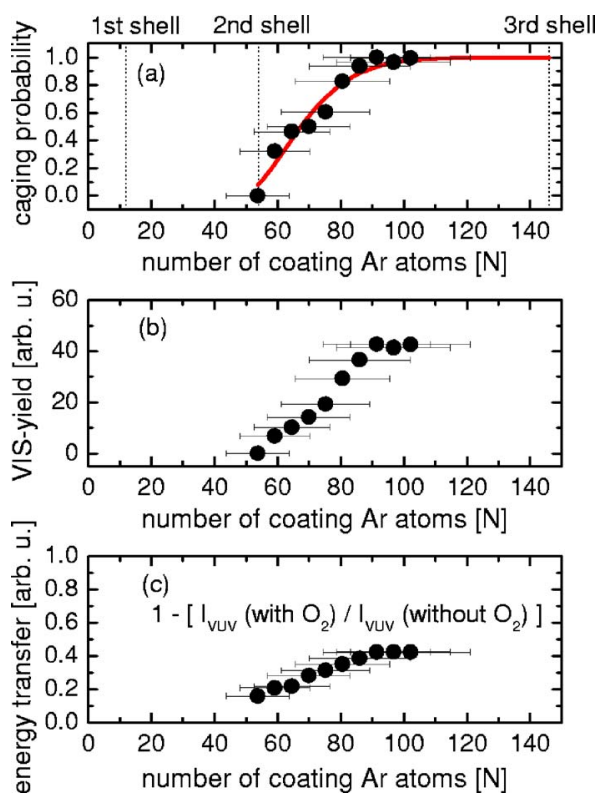

FIG. 6. (Color online) (a) Caging probability as a function of the number of coated Ar atoms $N$ surrounding the $\mathrm{O}_{2}$ molecule derived by normalization of the total vis fluorescence yield (b) to the experimentally determined energy transfer (c) (for details, see the text).

can extract the probability for a caging event as function of the number of coating $\mathrm{Ar}$ atoms around the $\mathrm{O}_{2}$ molecule. Quenching of vuv fluorescence indicates that energy is transferred from Ar to oxygen. Likewise the vis fluorescence is a measure for energy transfer from Ar to oxygen but also for the recombination of $\mathrm{O}$ atoms to ${ }^{3} \Delta_{u}$ oxygen or, for a smaller percentage, for the trapping of $\mathrm{O}$ atoms in $\mathrm{ArO}$ sites. One also has to keep in mind that the absorption cross section of Ar clusters depends sensitively on the cluster size $N$. Thus, to estimate the size-dependent energy transfer $T(N)$ from the photoexcited Ar cluster to the oxygen dopant, the total vuv fluorescence yield $I_{\text {vuv }}\left(\mathrm{O}_{2}\right)$ is normalized to the pure $\mathrm{Ar}$ cluster absorption $I_{\text {vuv }}$. Strong quenching of Ar fluorescence results in low $I_{\text {vuv }}\left(\mathrm{O}_{2}\right)$ signal which is equivalent to efficient energy transfer to embedded $\mathrm{O}_{2}$. The energy transfer efficiency $T(N)$ is described by

$$
T(N)=1-\frac{I_{\mathrm{vuv}}\left(\mathrm{O}_{2}\right)}{I_{\mathrm{vuv}}}(N)
$$

and plotted in Fig. 6(c). It can be seen that the energy transfer efficiency $T(N)$ is size dependent-an effect that we relate to the degree of excitonic delocalization. The excitonic delocalization increases with the number of Ar atoms and, as a consequence, the probability for spatial and energetic overlap with the oxygen wave function is increased. To derive the caging probability as a function of the number of deposited Ar atoms, the vis fluorescence yield [Fig. 6(b)] is weighted with respect to $T(N)$. The caging probability derived in this way is shown in Fig. 6(a).

According to this analysis, the caging probability drastically increases once 50-60 Ar atoms are deposited around the $\mathrm{O}_{2}$ molecule, approaching unity at around $100 \mathrm{Ar}$ atoms. What is the reason for obtaining exactly this number and how does it relate to the layer thickness? Small $\mathrm{Ar}_{N}$ clusters assume polyicosahedral structures with fivefold symmetry ${ }^{81}$ and exhibit closed shells with increased stability for $N$ $=13,55,147, \ldots$, atoms/cluster. ${ }^{82}$ If we assume that $\mathrm{O}_{2}$ monosubstitutes the central Ar atoms, as was shown theoretically for $\mathrm{F}_{2}$ in $\mathrm{Ar}_{54},{ }^{83}$ the experimental results in Fig. 6 show that by filling the first shell (dotted lines) photo dissociation is not affected. Moreover, our findings show that closing of the second icosahedral shells is necessary to cage the excited fragments effectively. The size of the embedded clusters depends on the Ne host cluster size distribution in the supersonic beam folded with the distribution derived from the Poisson statistics of the pickup process. A pickup pressure that leads to about 100 embedded $\mathrm{Ar}$ atoms is equivalent with all oxygen-doped clusters in the beam being codoped with at least $54 \mathrm{Ar}$ atoms. If two Ar layers were sufficient to induce recombination and vis luminescence of oxygen, then establishment of a third Ar layer will not increase the vis luminescence any further. Indeed, exactly this is what we observe: the saturation of vis fluorescence coincides with a pickup pressure for which all oxygen doped clusters have their second icosahedral Ar shell completed. The absence of a cage effect upon deposition of one cover layer suggests that the dissociation products are predominantly formed with rather high kinetic energy. This gives further evidence that vis light emitted from small embedded $\mathrm{Ar}_{N} \mathrm{O}_{2}$ clusters originates mainly from recombined excited oxygen dimers in the $A^{\prime}{ }^{3} \Delta_{u}$ state, where $2.3-3.5 \mathrm{eV}$ are released in the formation process rather than from ArO. On the other hand, it is known that the energetics and dynamics of dissociation and its suppression sensitively depend on the local environment of the dopant. Studies on $\mathrm{O}_{2}$ in crystalline $\mathrm{Ar}$ showed increased probability for permanent dissociation at defect sites, whereas substitutionally isolated $\mathrm{O}_{2}$ did not dissociate at excess energies $\leqslant 2.5 \mathrm{eV}$. ${ }^{3}$ The small embedded Ar clusters investigated in our work are likely to possess an amorphous structure and should therefore exhibit a large number of defect sites inherent to the formation process. This could explain to some extent the fact that the first 40-50 Ar atoms had almost no effect on the dissociation. Other groups observed signs of caging at much smaller shell thicknesses of Ar, ${ }^{20,21,25-28,30}$ but the molecules and the involved kinetic energies where different which makes a direct comparison difficult. Unfortunately, we could not record energy-resolved fluorescence spectra in the pickup experiment that varied the number of coating Ar atoms because the intensity was too low. Although the total vis signal should be dominated by the ${ }^{3} \Delta_{u}$ fluorescence, it would have allowed us to assess possible changes in the intensity ratio between the ${ }^{3} \Delta_{u}$ and the ArO fluorescence. By doing this it would have been possible to assess the kinetic energy dependence of the cage effect in much greater detail.

\section{CONCLUSION}

We have investigated the dissociation and recombination of photoexcited molecular oxygen isolated in rare Gas clusters with energy-resolved fluorescence excitation spectroscopy using monochromatic synchrotron light in the vacuum ultraviolet spectral range. The molecules were embedded inside Ar clusters using either the coexpansion or the pickup 
method. In particular, the effect of the number of coated $\mathrm{Ar}$ atoms around the $\mathrm{O}_{2}$ molecule, i.e., the cage thickness on the dissociation process, was studied.

Energy-resolved fluorescence spectra of $\mathrm{Ar}_{9700} \mathrm{O}_{2}$ produced by coexpansion of a dilute $\mathrm{Ar} / \mathrm{O}_{2}$ gas mixture showed electronically excited $\operatorname{ArO}\left(2{ }^{1} \Sigma^{+} \rightarrow 1{ }^{1} \Sigma^{+}\right)$and $\mathrm{O}_{2}\left(A^{\prime 3} \Delta_{u}\right.$ $\left.\rightarrow X^{3} \Sigma_{g}^{-}\right)$as photoreaction products. The fluorescence light was found to depend on the excitation energy showing that energy was transfered from Ar excitons to $\mathrm{O}_{2}^{+}$. The observed threshold showed that in Ar clusters the $\mathrm{O}_{2}^{+}$state is lowered in energy. The nonradiative decay along dissociative potential curves was found to populate electronically excited $\mathrm{O}\left({ }^{1} S+{ }^{1} D\right)$ or $\mathrm{O}\left({ }^{3} P+{ }^{3} P\right)$. The excited oxygen atoms recombined inside the argon cage which was detected by fluorescence from the $A^{\prime 3} \Delta_{u}$ state.

In a second set of measurements the doped clusters were prepared inside large neon clusters by a sequential pickup process of $\mathrm{O}_{2}$ molecules and argon atoms. Thereby, small embedded $\operatorname{Ar}_{N}$ clusters $(50 \leqslant N \leqslant 100)$ with $\mathrm{O}_{2}$ in the center were formed. Characteristic excitonic absorption bands of $\mathrm{Ar}$ clusters $(12.0-12.9 \mathrm{eV})$ were excited and the fluorescence was recorded in different wavelength regions. As an effect of the energy transfer to the oxygen the Ar cluster vacuum ultraviolet fluorescence was quenched. On the other hand, visible fluorescence was found to increase in intensity with growing thickness of the Ar layer. By analyzing the total fluorescence yield in the vacuum ultraviolet and visible spectral ranges as function of the number of coated Ar atoms, we derived that oxygen atoms with up to $3.5 \mathrm{eV}$ kinetic energy are completely caged by two solvation layers of Ar atoms.

\section{ACKNOWLEDGMENTS}

We are grateful to Steve Baker and Stephen Ball for helpful comments while preparing this paper. The financial support provided by the Deutsche Forschungsgemeinschaft under Grant Nos. Mo719/1-3 and LA 1431/2-1 is gratefully acknowledged. K.v.H. acknowledges funding by the British Council.

${ }^{1}$ A. V. Danilychev and V. A. Apkarian, J. Chem. Phys. 99, 8617 (1993).

${ }^{2}$ A. V. Danilychev and V. A. Apkarian, J. Chem. Phys. 100, 5556 (1994).

${ }^{3}$ A. V. Danilychev and V. A. Apkarian, Chem. Phys. Lett. 246, 139 (1995).

${ }^{4}$ A. V. Danilychev, V. E. Bondybey, V. A. Apkarian, H. Kajihara, S. Tanaka, and S. Koda, J. Chem. Phys. 103, 4292 (1995).

${ }^{5}$ V. A. Apkarian and V. E. Bondybey, Chem. Phys. 189, 137 (1994).

${ }^{6}$ V. A. Apkarian and N. Schwentner, Chem. Rev. (Washington, D.C.) 99, 1481 (1999).

${ }^{7}$ M. Gühr, M. Bargheer, M. Fushitani, T. Kiljunen, and N. Schwentner, Phys. Chem. Chem. Phys. 9, 779 (2007).

${ }^{8}$ J. Mestdagh, B. Soep, M. Gaveau, and J. Visticot, Int. Rev. Phys. Chem. 22, 285 (2003).

${ }^{9}$ B. Schilling, M. Gaveau, O. Sublemontier, J. Mestdagh, J. Visticot, X. Biquard, and J. Berlande, J. Chem. Phys. 101, 5772 (1994).

${ }^{10}$ C. Gée, M. Gaveau, O. Sublemontier, J. Mestdagh, and J. Visticot, J. Chem. Phys. 107, 4194 (1997)

${ }^{11}$ L. Moussavizadeh, K. von Haeften, L. Museur, A. Kanaev, M. Castex, R. von Pietrowski, and T. Möller, Chem. Phys. Lett. 305, 327 (1999).

${ }^{12}$ E. Lugovoj, J. P. Toennies, and A. Vilesov, J. Chem. Phys. 112, 8217 (2000).

${ }^{13}$ A. V. Kanaev, L. Museur, T. Laarmann, S. Monticone, M. C. Castex, K. von Haeften, and T. Möller, J. Chem. Phys. 115, 10248 (2001).
${ }^{14}$ A. Kanaev, L. Museur, F. Edery, T. Laarmann, and T. Möller, Eur. Phys. J. D 20, 261 (2002).

${ }^{15}$ A. Kanaev, L. Museur, F. Edery, T. Laarmann, and T. Möller, J. Chem. Phys. 117, 9423 (2002).

${ }^{16}$ A. Kanaev, L. Museur, F. Edery, T. Laarmann, and T. Möller, J. Chem. Phys. 120, 1801 (2004).

${ }^{17}$ T. Möller and G. Zimmerer, Phys. Scr., T T17, 177 (1987).

${ }^{18}$ J. Wörmer and T. Möller, Z. Phys. D: At., Mol. Clusters 20, 39 (1991).

${ }^{19}$ K. von Haeften, A. R. B. de Castro, M. Joppien, L. Moussavizadeh, R. von Pietrowski, and T. Möller, Phys. Rev. Lett. 78, 4371 (1997).

${ }^{20}$ B. Greenblatt, M. Zanni, and D. Neumark, Faraday Discuss. 108, 101 (1997).

${ }^{21}$ B. Greenblatt, M. Zanni, and D. Neumark, Science 276, 1675 (1997).

${ }^{22}$ Q. Liu, J. Wang, and A. Zewail, Nature (London) 364, 427 (1993).

${ }^{23}$ Q. Liu, J. Wang, and A. Zewail, J. Phys. Chem. 99, 11321 (1995).

${ }^{24}$ J. Wang, Q. Liu, and A. Zewail, J. Phys. Chem. 99, 11309 (1995).

${ }^{25}$ R. Baumfalk, N. Nahler, U. Buck, M. Niv, and R. Gerber, J. Chem. Phys. 113, 329 (2000).

${ }^{26}$ R. Baumfalk, N. Nahler, and U. Buck, Faraday Discuss. 118, 247 (2001).

${ }^{27}$ R. Baumfalk, N. Nahler, U. Buck, and S. Matter, Phys. Chem. Chem. Phys. 3, 2372 (2001).

${ }^{28}$ N. Nahler, M. Fárník, U. Buck, H. Vach, and R. Gerber, J. Chem. Phys. 121, 1293 (2004).

${ }^{29}$ U. Buck, J. Phys. Chem. A 106, 10049 (2002).

${ }^{30}$ U. Buck and M. Fárník, Int. Rev. Phys. Chem. 25, 583 (2006).

${ }^{31}$ S. Yang, S. Brereton, M. Wheeler, A. Ellis, and S. Matter, Phys. Chem. Chem. Phys. 7, 4082 (2005).

${ }^{32}$ S. Yang, S. Brereton, and A. Ellis, Int. J. Mass. Spectrom. 253, 79 (2006).

${ }^{33}$ A. Petrignani, W. J. van der Zande, P. C. Cosby, F. Hellberg, R. D. Thomas, and M. Larsson, J. Chem. Phys. 122, 014302 (2005).

${ }^{34}$ J. Frank and E. Rabinowitsch, Trans. Faraday Soc. 30, 120 (1934).

${ }^{35}$ X. Gu, D. Levandier, B. Zhang, G. Scoles, and D. Zhuang, J. Chem. Phys. 93, 4898 (1990).

${ }^{36}$ L. Perera and F. Amar, J. Chem. Phys. 93, 4884 (1990).

${ }^{37}$ V. Mozhayskiy, M. N. Slipchenko, V. K. Adamchuk, and A. F. Vilesov, J. Chem. Phys. 127, 094701 (2007).

${ }^{38}$ M. Lewerenz, B. Schilling, and J. P. Toennies, J. Chem. Phys. 102, 8191 (1995).

${ }^{39} \mathrm{~K}$. von Haeften, T. Laarmann, H. Wabnitz, and T. Möller, J. Electron Spectrosc. Relat. Phenom. 106, 199 (2000).

${ }^{40}$ R. von Pietrowski, M. Rutzen, K. von Haeften, S. Kakar, and T. Möller, Z. Phys. D: At., Mol. Clusters 40, 22 (1997).

${ }^{41}$ P. Slavicek, P. Jungwirth, M. Lewerenz, N. Nahler, M. Fárník, and U. Buck, J. Phys. Chem. A 107, 7743 (2003).

${ }^{42}$ M. Rutzen, S. Kakar, C. Rienecker, R. von Pietrowski, and T. Möller, Z. Phys. D: At., Mol. Clusters 38, 89 (1996).

${ }^{43}$ A. Lindblad, H. Bergersen, T. Rander, M. Lundwall, G. Öhrwall, M. Tchaplyguine, S. Svensson, O. Björneholm, and S. Matter, Phys. Chem. Chem. Phys. 8, 1899 (2006).

${ }^{44}$ M. Gaveau, E. Gloaguen, P. Fournier, and J. Mestdagh, J. Phys. Chem. A 109, 9494 (2005).

${ }^{45}$ H. Vach, J. Chem. Phys. 111, 3536 (1999).

${ }^{46}$ H. Vach, Phys. Rev. B 59, 13413 (1999).

${ }^{47}$ T. Laarmann, K. von Haeften, H. Wabnitz, and T. Möller, J. Chem. Phys. 118, 3043 (2003).

${ }^{48}$ D. Ievlev, I. Rabin, W. Schulze, and G. Ertl, Eur. Phys. J. D 16, 157 (2001).

${ }^{49}$ J. Wörmer, R. Karnbach, M. Joppien, and T. Möller, J. Chem. Phys. 104, 8269 (1996).

${ }^{50}$ T. Möller and G. Zimmerer, J. Opt. Soc. Am. B 6, 1062 (1989).

${ }^{51}$ R. Müller, M. Joppien, and T. Möller, Z. Phys. D: At., Mol. Clusters 26, 370 (1993).

${ }^{52}$ K. von Haeften, T. Laarmann, H. Wabnitz, and T. Möller, Phys. Rev. Lett. 87, 153403 (2001).

${ }^{53}$ J. Goodman and L. Brus, J. Chem. Phys. 67, 1482 (1977).

${ }^{54}$ M. Gudipati, F. Schouren, M. Kalb, and R. Wagner, Spectrochim. Acta, Part A 56, 2581 (2000).

${ }^{55}$ M. Lengen, M. Joppien, R. Müller, J. Wörmer, and T. Möller, Phys. Rev. Lett. 68, 2362 (1992).

${ }^{56}$ M. Lengen, M. Joppien, R. von Pietrowski, and T. Möller, Chem. Phys. Lett. 229, 362 (1994).

${ }^{57}$ R. von Pietrowski, K. von Haeften, T. Laarmann, T. Möller, L. Museur, and A. Kanaev, Eur. Phys. J. D 38, 323 (2006). 
${ }^{58}$ R. Karnbach, M. Joppien, J. Stapelfeldt, J. Wörmer, and T. Möller, Rev. Sci. Instrum. 64, 2838 (1993).

${ }^{59}$ O. F. Hagena, Z. Phys. D: At., Mol. Clusters 4, 291 (1987).

${ }^{60}$ U. Buck and R. Krohne, J. Chem. Phys. 105, 5408 (1996).

${ }^{61}$ J. Farges, M. F. de Feraudy, B. Raoult, and G. Torchet, Surf. Sci. 106, 95 (1981).

${ }^{62}$ S. Goyal, D. L. Schutt, and G. Scoles, J. Chem. Phys. 102, 2302 (1995).

${ }^{63}$ M. Lewerenz, B. Schilling, and J. P. Toennies, J. Chem. Phys. 102, 8191 (1995).

${ }^{64}$ I. Fugol, Adv. Phys. 27, 1 (1978).

${ }^{65}$ M. S. Gudipati, Chem. Phys. 201, 451 (1995).

${ }^{66}$ C. A. Long and G. E. Ewing, J. Chem. Phys. 58, 4824 (1973).

${ }^{67}$ K. von Haeften, T. Laarmann, H. Wabnitz, and T. Möller, J. Phys. B 38, S373 (2005).

${ }^{68}$ G. Herzberg, Can. J. Phys. 31, 657 (1953).

${ }^{69}$ H. Okabe, Photochemistry of Small Molecules (Wiley, New York, 1978).

${ }^{70}$ C. K. Rhodes, Excimer Lasers (Springer, Berlin, 1984).

${ }^{71}$ A. A. Radzig and B. M. Smirnov, Reference Data on Atoms, Molecules and Ions (Springer-Verlag, Berlin, 1985).

${ }^{72}$ S. L. Guberman, Science 278, 1276 (1997).
${ }^{73}$ K. von Haeften, A. Metzelthin, S. Rudolph, V. Staemmler, and M. Havenith, Phys. Rev. Lett. 95, 215301 (2005).

${ }^{74}$ T. Förster, Delocalized Excitation and Excitation Transfer (Academic, New York, 1965).

${ }^{75}$ A. Belov, I. Fugol, E. Yurtaeva, and O. Bazhan, J. Lumin. 91, 107 (2000).

${ }^{76}$ M. Gudipati and M. Kalb, Chem. Phys. Lett. 307, 27 (1999).

${ }^{77}$ T. Laarmann, A. Kanaev, K. von Haeften, H. Wabnitz, R. von Pietrowski, and T. Möller, J. Chem. Phys. 116, 7558 (2002).

${ }^{78}$ W. Stevens, Chem. Phys. Lett. 38, 374 (1976).

${ }^{79}$ T. Laarmann, K. von Haeften, A. Kanaev, H. Wabnitz, and T. Möller, Phys. Rev. B 66, 205407 (2002).

${ }^{80}$ A. Kanaev, L. Museur, F. Edery, T. Laarmann, and T. Möller, Phys. Rev. B 69, 125343 (2004)

${ }^{81}$ S. Kakar, O. Björneholm, J. Weigelt, A. R. B. de Castro, L. Tröger, R. Frahm, T. Möller, A. Knop, and E. Rühl, Phys. Rev. Lett. 78, 1675 (1997).

${ }^{82}$ M. R. Hoare, Adv. Chem. Phys. 40, 49 (1979).

${ }^{83}$ M. Y. Niv, M. Bargheer, and R. B. Gerber, J. Chem. Phys. 113, 6660 (2000). 